\title{
Enacting the Soft Automaton: Empirical Ontologies of Two Soft Robots from Technical Research and Media Art
}

\author{
Jonas Jørgensen \\ Digital Design Department \\ IT University of Copenhagen \\ Rued Langgaards Vej 7, DK-2300 \\ Copenhagen S, Denmark \\ jjoe@itu.dk
}

\begin{abstract}
This paper examines two soft robots from technical research and media art respectively and the practices through which they come into being. Departing from a juxtaposition of video presentations of the two robots, the empirical ontologies of a soft robot enacted in practice are analysed. The paper argues that two different versions of softness are being done and that the two sets of practices concomitantly respecify "knowledge" and "autonomy" as concepts, with different ethical and political implications.
\end{abstract}

Soft robotics. Soft material robots. Media art. Robotic art. Empirical ontology.

\section{INTRODUCTION}

In the course of the past ten years, soft robotics has become a thriving subfield of technical robotics research. Despite an observable tendency towards convergence, a number of different definitions of soft robotics and what a soft robot is still exist within the technical literature. Wang and colleagues (2017), for instance, remark that soft robotics "encompasses solutions that interact with [the] environment relying on inherent or structural compliance". Whereas Rus and Tolley (2015) define soft robots as "systems that are capable of autonomous behavio[u]r, and that are primarily composed of materials with [elastic] moduli in the range of that of soft biological materials".

Constructing robots from soft and elastic materials yields a number of technical benefits that include safe interaction through passive compliance, the possibility for shape-shifting and bodily adaptation, ease of control through morphological computation, and the potential to reuse stored elastic energy. But soft robots are equally endowed with a specific expressivity and a different aesthetic than their rigid precursors. These latter aspects have recently begun to be explored in a number of appropriations of soft robotics technology by artists, designers and architects. Examples of soft robots featured in artworks that were produced prior to soft robotics' emergence as a field, however, also exist.
This paper seeks to unpack the notion of a soft robot through an ontological mode of analysis inspired by Actor-Network Theory (ANT). Departing from the assumption that ontology, understood not as "that which is" but as "how things exist", is entwined with ethics and politics, the paper explores the practices of designing, constructing, interacting with and thinking about soft robots within two projects of technical robotics research and media art respectively. How are soft robots "being done" and by which means are different versions of soft materiality enacted? What tendencies and capacities of soft matter do these different soft robots actualise? In what sense is softness rendered active or agential?

\subsection{Approach}

As mentioned, the approach taken is inspired by ANT and the so-called ontological turn within Science and Technology Studies (STS). It is grounded in the argument put forth here that reality, as we inhabit it, is performed within different practices. Reality itself is thus multiple, not in a social constructivist sense, but in a deeper ontological sense (Mol 1999). And an object, such as a soft robot, can come in various versions, that despite sharing physical similarities, or even being physically identical, might diverge ontologically. In 
their studies of Atlantic salmon, John Law and Marianne Lien (2012) put this in relational terms as "different salmon are done in different practices" and "different salmon are being enacted". And Annemarie Mol (2014) has similarly articulated this notion in her argument that each version of practicing stages, performs, does, and enacts a different version of "the" object, which must therefore be considered "an object multiple" (Mol 2014). Within the ANT tradition this assumption of ontological multiplicity is operationalised in interpretive strategies that foreground and study practical ontologies empirically by paying close attention to the practices and networks of relations through which a specific version of an object is brought forth.

In the case of soft robotics as an emerging technology, the indeterminacy and multiplicity of the object in question, is perhaps even more readily apparent, as the technical object, a soft robot, is currently still being invented and articulated. But what a soft robot is and does, is also already gradually becoming more specified and stabilised through the contingent processes, actions, and discourses that make up practices. At this juncture, it is therefore relevant to consider how different enactments of "a soft robot" diverge, and that, given the choice between these different versions, to reflect on what politics and ethics that are enacted in conjunction with their differences.

The main interest of this paper is therefore to explore how soft robots get put together within two different contexts and associated sets of practices. Consequently, its focus lies on analysing the practices and the soft robot ontologies they enact how specific versions of "a soft robot" emerge and how these might differ. Furthermore, the paper seeks to engage with the merits and drawbacks of these two different ways of assembling "a soft robot". It aims to extend the specific risks and potentials "that singulari[s]e each position", to use a formulation of Isabelle Stengers (2005a).

\subsection{Analysis of video / analysis of practices}

A few further methodological remarks are needed before continuing, about how this paper extends the analytical practices of ANT and STS. Unlike most work anchored in the STS tradition, this paper is not based on ethnographic fieldwork. Instead it utilises two videos as its most immediate empirical materials. Even if ANT as a theoretical formation is critical towards the notion that there is ever such a thing as an unmediated access to the field, or reality as such, the kind of access to practices provided by a video obviously differs from the one obtained by being present at a physical site and associating with informants. Moreover, a framing evidently occurs when practices are translated into the video medium. Consequently, when analysing video, one obviously runs the risk of mistaking an edited representation for "the thing itself". In my analyses, I aim to stay aware of the limited and mediated access to practices the two videos offer. Both videos are clearly censored and staged accounts influenced by specific agendas and aspirations as well as the affordances of video as a medium. But analysing the practices as they are depicted in these videos rather than doing field ethnography, I argue, holds a different potential. Precisely because the videos are self-representations emerging from the practices they depict, what is included or excluded betrays assumptions about what is important and unimportant within these practices. Comparative analysis can thus function as a means to become aware of the blind spots of each video and potentially reconstruct some of what is left out in each of them. A further resource, that forms a backdrop for this work, yet remains a tacit voice in the text, is my own involvement with soft robotics, that have unfolded as both academic research and artistic practice, over the course of the past two years.

Embracing the situatedness of my own account, I have chosen to let the paper reflect its process of coming into being by starting the analysis with excerpts from notes that I originally jotted down while watching the videos on my computer as a prerequisite for writing this paper. Unless otherwise specified, quotations come from these notes.

\section{TWO VIDEOS}

An upbeat yet restrained music played on acoustic instruments is heard (it is similar to the kind of music that is featured in commercials for products). The first images we see is of the semitransparent Octobot with its characteristic blue and red fuel chambers and channels. It is seen from above on a white background. The title of the video then emerges in the white space next to the robot along with the Harvard seal ...

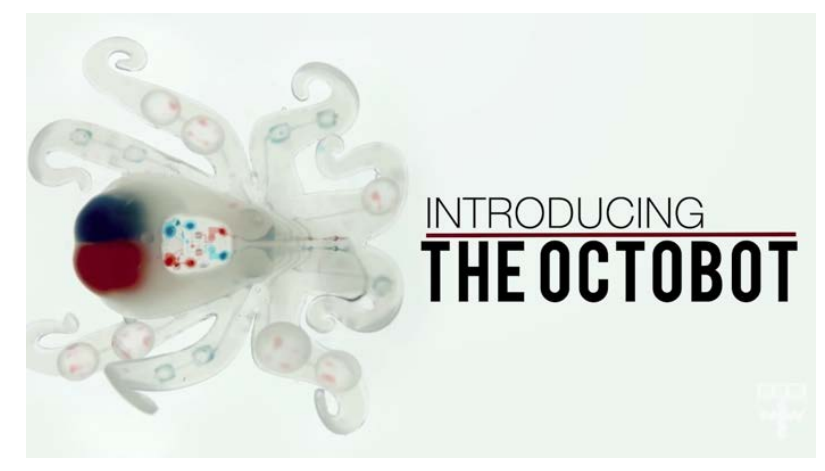

Figure 1: Still image from the opening shot of the first video. Courtesy of Harvard SEAS.

The first video (Harvard University 2016) disseminates research from an academic paper 
published as a letter in Nature, one of the most prestigious high impact science journals. The paper is authored by seven researchers from Harvard University and Weill Cornell Medicine. The video was produced by Leah Burrows, a Science and Technology Communications Officer at Harvard University. It was released on YouTube on 24 August 2016, the same day as the paper was published. The video is 1 minute and 34 seconds long and entitled "Introducing the Octobot".

The opening shot shows a woman sitting with a piece of paper reading aloud from it. Besides the voice only static noise and room noises can be heard. There is no professional lighting to improve the quality of the video images. The woman reads [...] in Spanish. There are English subtitles displayed at the bottom of the video. She informs us that she has designed "an autonomous robotic agent, whose most relevant behaviour is its capacity of having a body 'language'”.

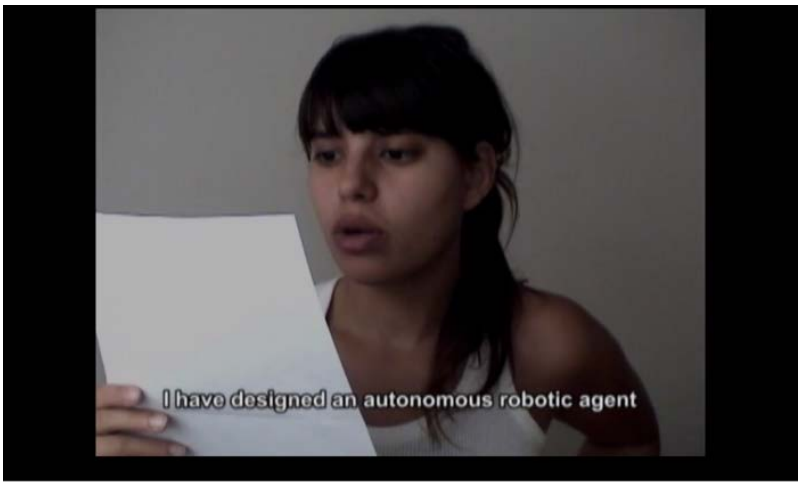

Figure 2: Still image from the beginning of the second video. Courtesy of Paula Gaetano Adi.

The second video (Gaetano Adi 2006) is stored on the Vimeo platform and embedded on the webpage of media artist Paula Gaetano Adi. It documents her robotic artwork Alexitimia (2006/2007). It was produced by the artist in 2006 and is 9 minutes and 43 seconds long ${ }^{1}$.

\subsection{Fabrication}

What defines a soft robot and through which methods and procedures might we know about and construct soft robots? Proceeding from an interest in empirical ontology, these are some of the questions we can ask of practices. For both videos, the answers given seem to hinge on revealing and making visible an origin and function but also on something more. Both videos share a similar symmetrical narrative structure: first there is footage of the robot, then its fabrication and functioning are explained and lastly the finished robot is shown again.

Consider the midsection of the Octobot video:
We see footage of the manufacturing of the robot, which occurs on a multimaterial embed 3D printer that uses a syringe to pierce through the uncured liquid silicone in a mould. Materials with the right mechanical properties are deposited into [what]... will later become the body of the robot. The [rigid] robot constructing the soft robot moves fast and precisely ... The uncured silicone lights up in a bright fluorescent bluish hue, and the printed inner channels appear in red. ... The [soft] robot is then seen from above, as in the beginning, and we are told that it is controlled with a microfluid[ic] logic circuit ... "The logic circuit acts just like a circuit board, autonomously directing fuel"

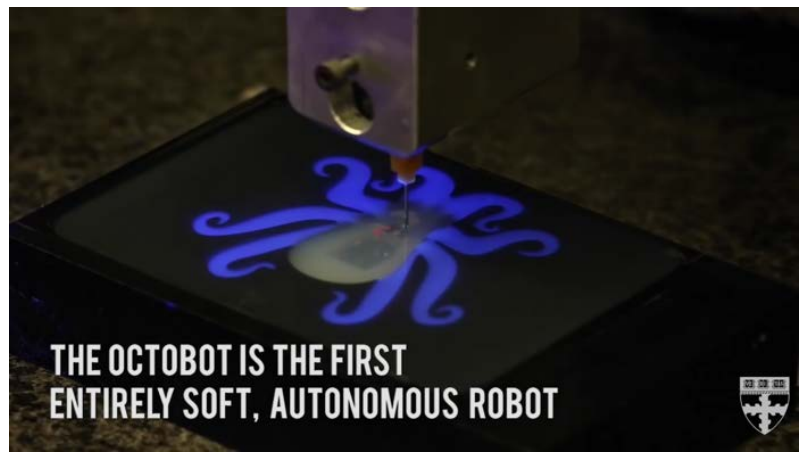

Figure 3: Still image from the video showing the Octobot's fabrication. Courtesy of Harvard SEAS.

Compare this with how the fabrication is depicted in Gaetano Adi's video:

4:40: A spherical shape is shown occupying most of the frame. She gradually adds pieces of clay to cover its surface, making sure to even them out using both of her hands ... A gloved hand is distributing a white layer of liquid material (latex) across the surface. Footage where hoses are seen being attached. This occurs with the use of a brush that gently adds latex on top of a white fabric mesh that looks like gauze ... Flexible piezo film sensors are added, painted over with latex meticulously (... [she] handles and controls the brush skilfully) to sense the touch. 6:23: Using what looks like a soldering iron she very carefully punches holes [in the tubing], wearing glasses to better see ... The gestures and setup look like an operation being performed on a human body that is partially covered, but the tool that is used is usually used to repair or assemble electronics. It is at once a technical operation (repairing a machine) and a surgery... 


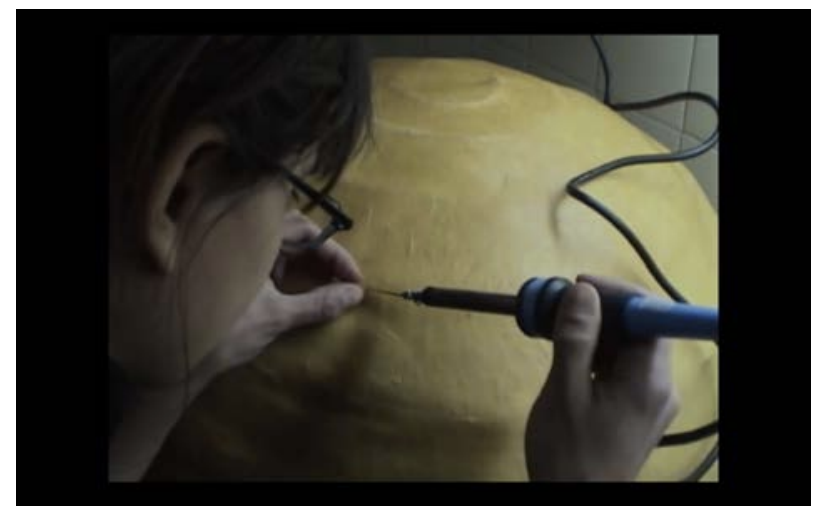

Figure 4: Still image from the video: Holes are made. Courtesy of Paula Gaetano Adi.

6.47: The semi-sphere is seen lying on a plinth(?) it is lifted over to reveal wires, which the woman is seen twisting together (the sensors were "connected in parallel..."). The microcontroller is placed in a small plastic suitcase-like enclosure that looks to be fixed atop a baking tin. It activates four pumps, hidden inside [the] pedestal in [a] reservoir with distilled water.

Juxtaposing the two different versions of soft robot fabrication in the two videos, it becomes clear that soft robots are enmeshed in dissimilar practices characterised by markedly different procedures and aspirations. In the Octobot video, there are no humans present. We are in a sort of ideal decontextualised flat space of modern science, devoid of subjects and subjectivities. The only actors present are precise machines that manipulate and combine materials with full reproducibility. In great contrast to this, Alexitimia is always depicted in the presence of its human creator, the artist, whose physical labour is portrayed as bringing it into existence through careful and caring attention.

\subsection{Production as knowledge}

How to make sense of these differences? Scale obviously has a bearing on some of them - the Octobot is driven by chemical reactions and microfluidic circuits whereas Alexitimia has a size comparable to that of the human body and is powered by a traditional microcontroller, electrical sensors and water pumps that can be assembled by hand. The two videos thus attest to what soft elastomers are capable of becoming when entering into composition with computing technologies on different scales. But the actions and practices depicted also reverberate with ideals from their respective cultural domains - the artwork as a materialization of the artist's embodied mastery of her physical medium and the scientific object as founded on pure objectivity, unsoiled by human desire. The two fabrication processes can, moreover, be seen to echo different historical modes of technical practice - automated industrial mass production in the case of the Octobot and traditional crafts in the case of Alexitimia. This observation that the Octobot fabrication takes mass production as its ideal might also lead one to take note of the word "Veritas" that is included in the diagram of the Octobot's microfluidic logic circuit in the video (Figure 6) and printed on the physical circuit, just as a brand name often is on mass produced commodities.

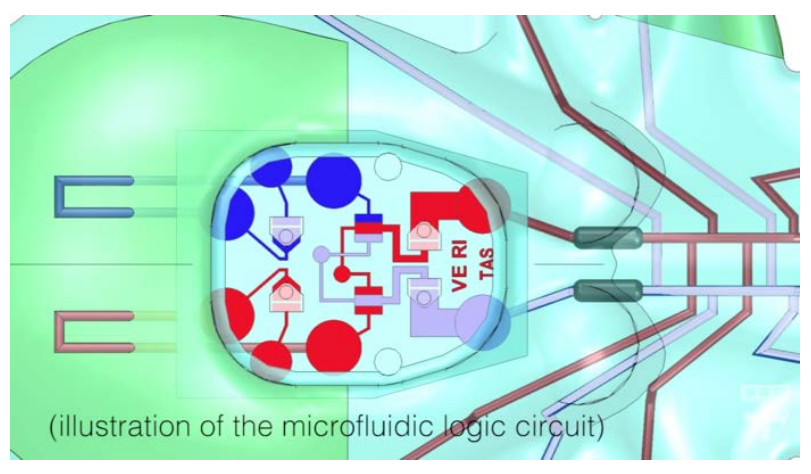

Figure 5: Still image from the video: Diagram of the logic circuit. Courtesy of Harvard SEAS.

"Veritas", truth, is the motto of Harvard, its brand. As a sign, "Veritas" branded on the robot performs several functions. On the one hand, it is an authoritative sign of truth, that is - a symbol referring to Harvard and the Ivy League system and its institutionalised politics of knowledge. But it also denotes the robot as a physical instance of the truth, a research insight. But how exactly does a thing become truth? And if this thing is the truth, then has this truth been produced, and not discovered? And how exactly did that occur?

If one looks elsewhere than the video, it becomes clear that this process was indeed a production, and one of a considerable volume. More specifically, 300 not-quite Octobots were produced before arriving at a functional Octobot (Sklar 2016). That this iterative material process was needed to produce the truth of the Octobot, points to that soft materials are hard to simulate accurately in a computer. While roboticists routinely simulate rigid morphologies to determine their most optimal designs, this is not easily done with complex soft morphologies with existing techniques such as the finite element method $(F E M)$. In this respect, the dynamics of soft materials can be said to resist or escape numerical representation and computation as a force of knowledge production. This unknowability of the physicality of soft materials exemplifies what Andrew Pickering (1994) has termed the "resistance" of matter: When we probe and prod it, that is, when we push, it also pushes back in its own specific, and sometimes unpredictable, ways (see also Hayles 2014 on this). The right design parameters of a functioning Octobot could not be predicted beforehand by combining existing models from soft matter physics and chemistry. It had to 
emerge from complex dynamics and adjustments that were negotiated through material practice.

\subsection{Unknowing softness}

The way Gaetano Adi stages and addresses her robot also points to something that is unknowable in the abstract. But here the notion that some insights about soft robots and soft materiality can only be experimented, or intuited, is embraced as an explicit and integral part of practice and the resulting artwork, and not merely seen as an obstacle to overcome. Overall, Alexitimia is concerned with trying to find a "body language of robots", Gaetano Adi tells us in the video, and with establishing "a dialog between two kinds of bodies: a corporal dialog" (Gaetano Adi 2006):

\begin{abstract}
the interface of the work is the skin, the sense of touch is what enables the interaction, and between them [the human and the robot] it is the sweating of the robot's body [t]hat creates the dialog. (Gaetano Adi 2006)
\end{abstract}

Despite the fact that the haptic exchange, described above, serves as the locus of the artwork, no attempt is, however, made to explain or interpret its content in the video. The technical functioning of Alexitimia is demonstrated in great detail, but the practices depicted before and after this propose that this is not all that there is to know about the robot. Presented in its partially assembled or unfinished state, the robot is rendered comprehensible to causal thinking and reasoning. But in the depiction of the interaction between the finished robot and the human, the emergence of a knowledge or even a not knowing (see Borgdorff 2012), that cannot be subsumed under the concept, appears to lie at the heart of the practices through which the robot's "functioning" is proposed to unfurl. In the video, we thus encounter multiple, yet specific, ways of interacting with Alexitimia through touch:

8:39: A hand is seen gently massaging the blob, which secretes liquid. Zoom out: The hand belongs to the creator. She is kneeling beside the robot

And different choreographies of touching unfold: a slow gentle caress, a surface smear, a mechanical folding of layers, an assembling of the skin-like material. The robot responds by sweating but also with a distinct vocabulary of bodily gestures afforded by its elasticity and structural configuration: bending in, popping back out immediately or slowly, folding inwards, lying in wait of what's next. The robot's response to the touch engenders the next touch, which engenders another response from the robot and so on, it seems. A sense of reciprocity emerges.

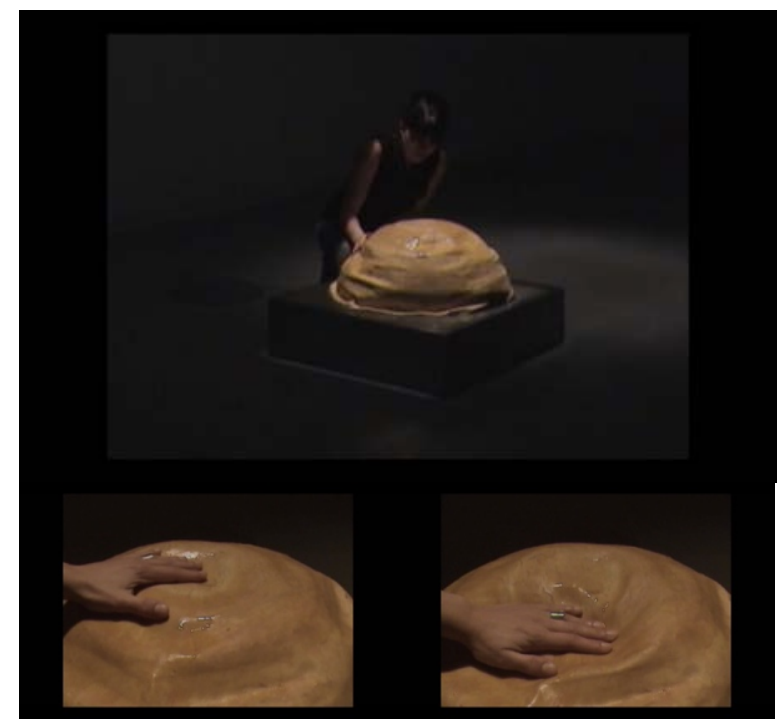

Figure 6: Still images from the video: Touching unfolding between the artist and the robot. Courtesy of Paula Gaetano Adi.

From the perspective of intentionality, touch might be considered the primary gesture through which softness becomes active or known. Yet Erin Manning has also articulated touch as a movement towards the not yet known (Blackman 2009). Specific gestures of touch, however, also entail an anticipation of a specific sensation and sometimes also an intent of use. Thus the repertoire of human gestures performed onto the robot can equally be considered embodied techniques that are informal ways of knowing softness. They each subtly index classes of activities that unfold within different contexts of human activity. Some are appropriate and fit for touching a material, others a machine, others still for engaging with a living being. Yet they all unfold slowly and exude a certain calmness and gentleness.

\subsection{Autonomy}

Given the videos' thorough dissection and demystification of the workings of both robots, it might seem curious that the discourse in both videos explicitly mention and emphasise "autonomy" - a concept that broadly refers to independent, selfconscious self-determination and not deterministic processes. Alexitimia is presented as "an autonomous robotic agent" (Gaetano Adi 2006) and the Octobot as "the first entirely soft, autonomous robot" (Harvard University 2016). Autonomy is of course a concept that in general figures prominently in robotics discourses. It relates to agency, or, one might say, the degree to which a robot is able to enact itself. But how and in what sense does softness afford autonomy for these two robots?

In The Robotics Primer, an introductory textbook on robotics, a robot is defined as: "an autonomous system which exists in the physical world, [that] can sense its environment, and can act on it to achieve 
some goals." And autonomy is further specified in the following manner: "An autonomous robot acts on the basis of its own decisions, and is not controlled by a human" (Mataric', 2007: 2). By this definition (which is of course itself rife with ambiguities) neither robot would obviously qualify as being autonomous - the Octobot does not have sensors and Alexitimia appears to be a simple reactive robot, only capable of one behaviour, namely to pump "sweat" when bending is detected by the piezo sensors above some threshold.

The Octobot is, however, also not conceived as autonomous by this broad textbook definition, but more narrowly by not being tethered to a pneumatic or electrical power source, as soft robots usually are. Hence, it is solely by virtue of breaking with the contingent assumption of contemporary soft robotics research that a soft robot must be tethered in order to function, that the researchers claim to have attained autonomy in the Octobot (Wehner et al. 2016). As mentioned already, the video emphasises this notion of autonomy-as-disconnection by depicting the robot separately from humans and any specific and identifiable environment. The manual human labour and handling involved in its production is also elided and its fabrication presented as fully automated - thus figuratively fulfilling the future vision to which the research project itself aims to contribute. Yet upon further inspection, it becomes evident that the humanless staging in the video, in fact conceals that the human sensorium and cognizing is deeply imbricated in the design and presentation of this "autonomous" robot. The footage of its manufacture and functioning, for instance, is played at ten, twelve and fifteen times the actual speeds at which they occur. And the colourful materials have been stained with pigments in order to better reveal the design and the functioning of the robot (Science Magazine 2016). Both the robot itself and its documentation have thus been adjusted and enhanced to lend themselves easily to the human eye and its temporalities and to be fully legible. Furthermore, as a research robot the Octobot is not intended to be a working robot that successfully fulfils some purpose "in the wild", it is only a "proof of concept" (Burrows 2016). Paradoxically, its primary function is therefore not to operate autonomously on its own but to perform and communicate itself as a research finding to a human audience.

In stark contrast to the Octobot, Alexitimia enacts a version of autonomy that seems only actualisable in the close company of a human. Its autonomy is equally not predicated on an ability to physically influence its environment in order to attain some specific goal. Instead it seems to be relational in kind and to inhere in the robot's proposed ability to sustain what Don Ihde (1990) refers to as an alterity relation, that is, to conjure up a sense of independent existence or quasi-otherness (whose nature and implications I will return to in the final subsection of this paper).

\section{SOFTNESS AS A FORCE OF ROBOTICS}

So what is at stake politically in the two different enactments of a soft robot? The analysis unfolded so far suggests that central categories of soft robotics can in themselves become pliable and malleable in practices: Softness and knowledge hereof can be constructed in more than one way, soft autonomy equally so. But this should not make us forget that how things exist, and whose reality is considered more accurate is indeed a political question. Domination could in fact be considered "a matter of holding the capacity to differ under control", as anthropologists Martin Holbraad, Morten Axel Pedersen and Eduardo Viveiros de Castro have put it (Holbraad et al. 2014). As a first move in approaching the politics of these two robots, we might therefore start by taking note of the different positions of enunciation from which they are articulated. The Octobot was published in Nature and has been hailed widely as a seminal research accomplishment in soft robotics research. Alexitimia and other soft robotic artworks, however, are yet to be recognised as contributions to the production of knowledge about soft robots.

To go further in addressing this question, it is insightful to dwell on another way in which specific object ontologies enact an ontological politics, namely through their reality effects and concomitant modulations of other objects and concepts (Mol 1999). I have already noted how "softness", "knowledge" and "autonomy" are respecified in different ways within the two sets of soft robotic practices. That is, these concepts are enacted with specific meanings that modulate their theoretical definitions (which in turn might lead us to reconsider these $)^{2}$. A central contrast herein is that the practices that envelop the Octobot tend to emphasise a separation between humans and robots, while those of Alexitimia embrace the similarities and the zone of indiscernibility softness can produce. And through this, the two robots enact two different ways of distinguishing between a human and a robot, and with that two different ways of performing human subjectivity, different modes of subjectivation, that have purchase on politics and ethics. To be more precise, the Octobot research was driven by, so it appears, an instrumental desire for humans to gain control of soft matter. And this matter was to be mastered through practices that combined techniques of both modern science and cybernetics - measurements, numerical representation and abstraction ("Veritas", tellingly, resided in the robot's its "logic circuit" and not its limbs) and systematic technology-aided trial and 
error. A number of philosophies of technology and science have been critical of this set of epistemological operations, that have been considered "reductive" acts of epistemic violence towards situated experience and non-hegemonic ways of knowing. But seen from a post-critical perspective, the reduction they entail is not necessarily simply negative or lamentable. Concepts and abstractions, in general, function to reduce and manage the multitude of differences we encounter in the world. As such, they effectuate a reduction of complexity that is necessary for thinking and acting to occur. Hence in practice the reduction performed via natural science and technology is also productive. Furthermore, from a posthumanist perspective, Alexitimia's enactment of softness in a robot can equally be considered both reductive and productive, albeit in a different manner: It is predominantly articulated from the point of view of a human body and does not acknowledge the equally (possibly) real myriad processes that unfold through other modalities than human sensation on different scales (the global circuits of latex manufacture and their ecological and social effects, the entropic grounds of soft polymer elasticity etc.).

In line with this view of reduction as productive, Isabelle Stengers (2005b) has argued that instead of seeing the successes of experimental science and technology as instances of matter's submission to thought, we might also affirmatively view them as increases of what has now become possible: "[W]hat they [experimental science and technology] address becomes able to do what it could not do in the usual circumstances" (Stengers 2005b). Consequently, what we are witnessing in functioning technology, according to Stengers, is not submission but "a force which has been both unfolded and re-folded" (ibid.). So how is softness produced here, within these specific empirical ontologies of the two soft robots, how is softness actualised as a force and what does it become capable of?

By looking at soft matter and chemistry through concepts previously used to describe electronic circuits and aided by rapid prototyping technologies, it was possible to construct an oscillatory control circuit for the Octobot out of soft matter and chemical reactions. That is, coupled with these specific concepts and their associated practices and this equipment, softness can afford a robot untethered autonomy and allow for an integration of systems and components (actuation, control, computation) that are usually separate in a robot.

Alexitimia, on the other hand, evinced that assembled with a microcontroller and sensors as an interactive artwork, soft matter can equally engender what we might term "soft interactions" with humans. These exchanges were characterised by specific speeds and specific gestures that appear to be tied to soft matter in general (as previously described). The result was, that in this configuration, coupled with this set of practices, the physical softness of the material was translated into "soft" movements and also aided in manifesting other meanings that the word "soft" has as an adjective - "producing agreeable ... sensations", being "characterized by ease and quiet enjoyment" and being "of a calm or placid character" (Oxford English Dictionary).

\subsection{Towards an affirmative soft robot ethics}

That multiple meanings of softness were actualised in the "soft interactions" of Alexitimia, illustrates the need to go beyond the strictly technical definitions of softness, used within contemporary soft robotics research, when conceptualising the interactions that a soft robot might have with organisms or other elements in its environment. Furthermore, it points to the potential for artworks to help in inventing new ways of knowing and experiencing softness and a more adequate expanded vocabulary to address it.

Taken as a representation of an epistemic practice, the video on Alexitimia aligns with Andrew Pickering's description of how some interactive artworks can be considered "implicit invitation[s] ... to adopt a non- or post-scientific worldview which sees humanity not in a position of cognitive control but rather as simply caught up in the weather of unpredictable becoming" as he puts it (Pickering, 2016). In that sense Alexitimia's versioning of softness can be seen to point beyond a purely instrumental notion of technology, and towards a receptivity to not just the quasi-otherness of a soft artificial embodied agent, but also to the otherness that resides within the human, i.e. the non-human constituents of human subjectivity that elide conscious knowledge and control. Given the imperative of posthumanist theory to rid ourselves of the humanist fantasy of the Cartesian subject as autonomous and set above the world (see Braidotti 2013; Wolfe 2009), this is arguably a both relevant and timely experience. The work can, moreover, serve as a productive counter to the currently existing discourse on soft robot ethics. The only academic publication that currently deals with soft robots from the point of view of ethics namely worries that their soft tactility might have the effect of creating more deeply felt emotional attachments in interactions with humans. The authors therefore recommend soft roboticists who are involved in designing soft robots for interaction with humans to "balance tactile engagement against emotional manipulation" and to "model intimacy on the bonding with a tool not with a person" (Arnold and Scheutz 2017). This recommendation, formulated from a stance of morality (what people should do) rather than ethics (what people can become capable of), I posit, is problematic as it elides the growing body of 
work within STS and related disciplines that stress that advanced technological "tools" are rarely uncomplicated and straightforward to "bond with" let alone implement in societies. As I have tried to articulate through my treatment of the Alexitimia video, embracing, rather than repressing, the affective potentials of soft robots on the contrary has the potential to make us question the conception of robots as simple tools that we can expect to yield and fully control. Artworks and the onto-epistemic practices of art could potentially add this more modest way of engaging with and thinking about the world, that does not presuppose a fixed power relation between humans and world or humans and robots, to the current repertoire of methods within soft robotics research. If the proposed application of soft robots (outside the lab and the white cube) in so-called welfare and care technologies stands to happen, surely this would be no small feat, as this clearly raises other issues than functionality and safety, that one needs to attend to in a careful, experimental manner.

\section{REFERENCES}

Arnold, T. and Scheutz, M. (2017) The Tactile Ethics of Soft Robotics: Designing Wisely for HumanRobot Interaction, Soft Robotics, 4(2), pp. 81-87. doi: 10.1089/soro.2017.0032.

Blackman, L. (2009) 'Starting Over': Politics, Hope, Movement, Theory, Culture \& Society, 26(1), pp. 134-143. doi: 10.1177/0263276408099019.

Borgdorff, H. (2012) The Conflict of the Faculties. Perspectives on Artistic Research and Academia. Leiden: Leiden University Press.

Braidotti, R. (2013) The Posthuman. Polity, Cambridge, UK; Malden, MA, USA.

Burrows, L. (2016) The first autonomous, entirely soft robot, Available from: https://www.seas.harvard.edu/news/2016/08/firstautonomous-entirely-soft-robot (retrieved 22 June 2018).

Gaetano Adi, P. Alexitimia. Vimeo Available from: http://paulagaetanoadi.com/works/alexitimia/ (retrieved 30 May 2018).

Harvard University. (2016) Introducing the Octobot. Harvard University, YouTube Available from: https://www.youtube.com/watch?v=1vkQ3SBwuU4 (retrieved 30 May 2018).

Hayles, N. K. (2014) Speculative Aesthetics, Speculations: A Journal of Speculative Realism V, pp. 158-179.

Holbraad, M., Pedersen, M. A. and Castro, E. V. de (2014) The Politics of Ontology: Anthropological Positions - Cultural Anthropology. Available at: https://culanth.org/fieldsights/462-the-politics-of- ontology-anthropological-positions (retrieved 14 June 2018).

Ihde, D. (1990) Technology and the Lifeworld. Bloomington and Indianapolis: Indiana University Press.

Law, J. and Lien, M. E. (2013) Slippery: Field notes in empirical ontology, Social Studies of Science, 43(3), pp. 363-378. doi: 10.1177/0306312712456947.

Mataric', M. J. (2007) The Robotics Primer. The MIT Press, Cambridge, Massachusetts; London, UK.

$\mathrm{Mol}$, A. (1999) Ontological politics. A word and some questions, The Sociological Review, 47(S1), pp. 7489. doi: 10.1111/j.1467-954X.1999.tb03483.x.

Mol, A. (2014) A reader's guide to the "ontological turn" - Part 4. Available at: http://somatosphere.net/2014/03/a-readers-guideto-the-ontological-turn-part-4.html (retrieved 14 June 2018).

Oxford English Dictionary. Available at: www.oed.com (retrieved 14 June 2018).

Pickering, A. (1994) The Mangle of Practice. In: Scharff, R. C. and Dusek, V. (eds.) Philosophy of Technology: The Technological Condition: An Anthology (2014), 2nd ed. West Sussex: John Wiley \& Sons. pp. 260-265.

Pickering, A. (2016) Art, Science and Experiment, MaHKUscript. Journal of Fine Art Research, 1(1). doi: $10.5334 / \mathrm{mjfar} .2$.

Rus, D. and Tolley, M. T. (2015) Design, fabrication and control of soft robots, Nature, 521(7553), pp. 467-475. doi: 10.1038/nature14543.

Science Magazine (2016) 'Octobot' is the world's first soft-bodied robot. Science Magazine, YouTube Available from: https://www.youtube.com/watch?v=CDohWwEXQ6 8 (retrieved 30 May 2018).

Sklar, J. (2016) Meet the World's First Completely Soft Robot. MIT Technology Review Available from: https://www.technologyreview.com/s/603046/meetthe-worlds-first-completely-soft-robot/ (retrieved 30 May 2018).

Sormani, P. et al. (2017) Ethnomethodology, Video Analysis, and STS. In: Felt, U. et al. (eds.) The Handbook of Science and Technology Studies (2017), 4th ed. Cambridge and London: The MIT Press. pp. 113-137.

Stengers, I. (2005a) Beyond Conversation: The Risks of Peace. In Keller, C. and Daniell, A. Process and Difference: Between Cosmological and Poststructuralist Postmodernisms, SUNY Press, Albany. 
Stengers, I. (2005b) Introductory Notes on an Ecology of Practices. Cultural Studies Review, 11(1). pp.17-26.

Wang, L., Nurzaman, S. G. and lida, F. (2017) SoftMaterial Robotics, Foundations and Trends $₫$ in Robotics, 5(3), pp. 191-259. doi: $10.1561 / 2300000055$.

Wehner, M. et al. (2016) An integrated design and fabrication strategy for entirely soft, autonomous robots, Nature, 536(7617), pp. 451-455. doi: $10.1038 /$ nature19100.

Wolfe, C. (2009) What Is Posthumanism?. University of Minnesota Press, Minneapolis.

\section{NOTES}

1. Paula Gaetano Adi, email to author, 29 May 2018.

2. On the concept of respecification see Sormani et al. 2017. 